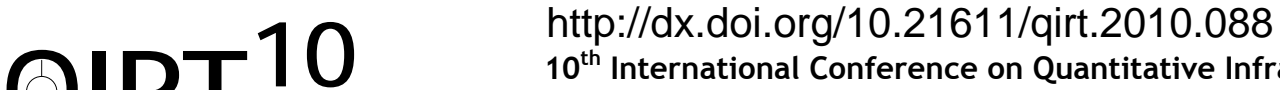 \\ $10^{\text {th }}$ International Conference on Quantitative InfraRed Thermography \\ July 27-30, 2010, Québec (Canada)
}

\section{Infrared thermography of solid surfaces under fire}

\author{
by J. Meléndez*, A. Foronda*, J. M. Aranda*, F. López* and F. J. López del Cerro** \\ *Dept. de Física, Universidad Carlos III de Madrid, 28911 Leganés (Madrid), Spain, melendez@fis.uc3m.es \\ **Laboratorios de Sistemas, Airbus Military, 28906 Getafe (Madrid), Spain
}

\begin{abstract}
Measurements in fire resistance tests of materials face two main difficulties: the harsh environment and the lack of spatial resolution of thermocouples (TCS). This paper presents a way to overcome these problems by using an infrared (IR) camera to study the exposed side of composite plates exposed to fire. A method to minimize the flame effect was developed, resulting in surface temperature maps. Errors in temperatures were studied, and results were compared to those of TC. It was found that the IR method provides better results, not only due to spatial resolution but also because of the non-intrusive nature of IR thermography, as opposed to the local effects caused by TCs.
\end{abstract}

\section{Introduction}

A fire is a rapidly changing, high temperature phenomenon inhomogeneus in space, and therefore fast, remote, 2-D measurements are particularly useful in fire studies. IR imaging has become a common technique, for instance, in the study of forest fires [1]. However, it faces difficulties when it comes to providing quantitative temperatures in fire environments. The flame gases have a transmittance (and therefore, emissivity) generally unknown that depends on concentration and has strong spectral features. Therefore, when imaging a flame the temperature readout of an IR camera is not indicative of the real object temperature. The first attempts to measure thermographically the temperature of a solid wall viewed through a flame used a spectral region $(10.6 \pm 0.5 \mu \mathrm{m})$ where the emission from $\mathrm{CO}_{2}$ and $\mathrm{H}_{2} \mathrm{O}$ is negligible, and, for thin flames, soot emission can be also neglected [2]. However, this is not true for other experimental conditions, and a procedure to discount flame contribution must be established. Pilch et al [3] practised a hole in the sample in order to have a region were signal is due only to soot radiation, and subtracted this from the sample image. This procedure is conceptually straightforward, but in most cases it is not possible to make a hole in the sample. In addition, the flame contribution to radiation depends on the position, and may be very different from that measured at the hole.

In this work we propose a thermographic method to measure temperatures of solid surfaces subject to a fire, which accounts for the flame contribution with no need for specific sample preparation. A "flame image" is obtained that is subtracted from the scene image to obtain a corrected image that is an approximation to the radiance emitted by the solid phase, and which can be translated into an instantaneous temperature map of the surface. Our method has been applied to the study of the temperature distribution in plates of composite materials subjected to fire resistance tests. It must be pointed out, however, that it is not a solution to the general problem of measuring temperatures of solid phases exposed to fire, since, as will be explained, it requires that flame is stable during the whole test, and that it is turned on and off very fast. These features apply to our experiment, but not to most fire situations.

\section{Experimental setup}

Experimental measurements on carbon fibre reinforced polymer plates were performed in a scaled-down reproduction of the plates' fire resistance certification test. A small diesel-fuelled burner was used to reproduce the flame produced by the standard burner used in certification testing, which is considerably larger. Flame develops very fast (in $t<0.1$ s) at the start up, it remains constant during the experiment, and its termination is instantaneous upon loss of control power, as a solenoid-operated fuel admission valve closes. Figure 1 shows two photographs, visible and infrared, of the setup during a fire test.

All plates used in the experiment were of the carbon fibre reinforced polymer (CFRP) kind. Plates were roughly square in shape, approximately $350 \times 350 \times 3 \mathrm{~mm}$. They were exposed to the burner flame outdoors, prior to any measurement, in order to liberate volatile compounds from the outermost layers, thus reducing smoke in the laboratory tests. Burning the surface is also useful in order to increase its emissivity, since soot has a large emissivity ( $\varepsilon=0.95 \pm 0.03$ according to [4]) that increases the baseline emissiviy of the undamaged surface of CFRP, estimated as $\varepsilon=0.80 \pm 0.01$ by Starnes et al [5].

A FLIR Thermovision A40M camera was used for measurements. This is a $320 \times 240$ focal plane array (FPA), uncooled microbolometer device with a spectral range of 7.5 to $13 \mathrm{um}$. 
The camera was connected via its Firewire interface to a PC-Windows platform running LabVIEW 7.1 software. A set of applications were developed to control image acquisition and perform processing. Typically, sequences 5 minutes long were taken at 20 frames per second (FPS), resulting in 6000 frames. These included flame startup and extinguishment and sometimes the cool-down period. After the tests, sequences were processed in a semi-automatic fashion. Figure 2 summarizes the processing scheme.
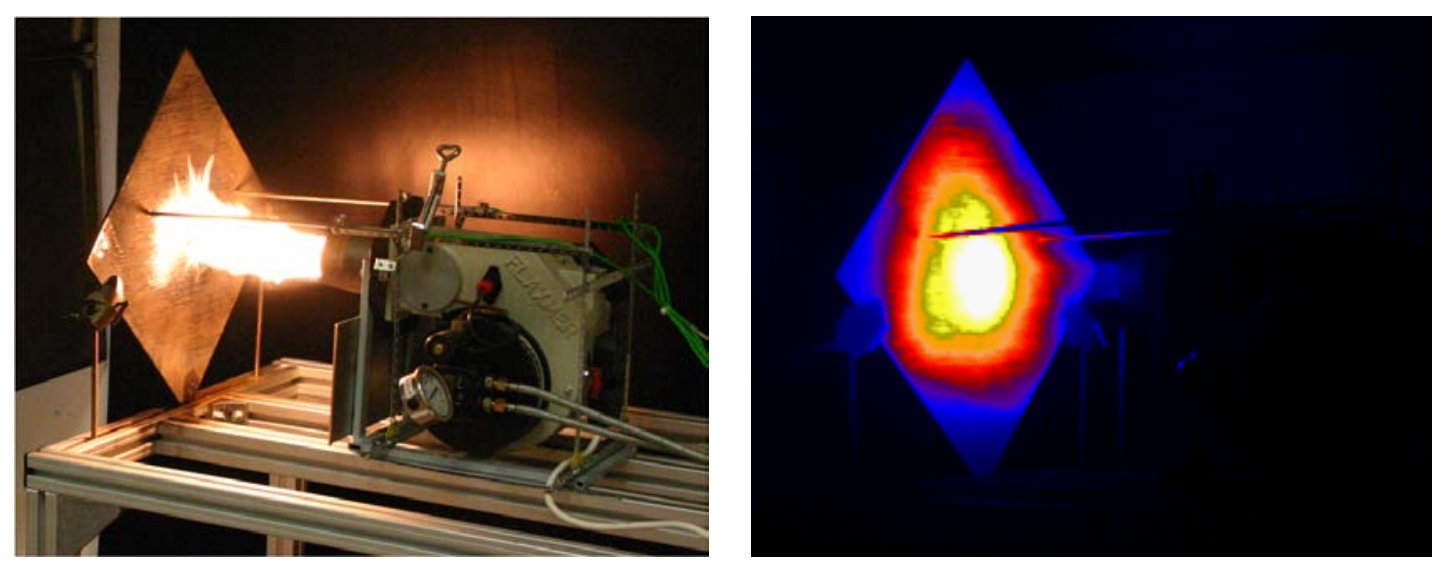

Fig. 1. Left: the experimental setup in operation during a fire test. The plate and the burner are supported on a frame built with aluminum profiles. Two thermocouples are placed in contact with the plate, fastened to the burner. Right: the same test viewed in IR, with a colour palette to improve visualization. Thermocouples are seen as two nearly horizontal lines.

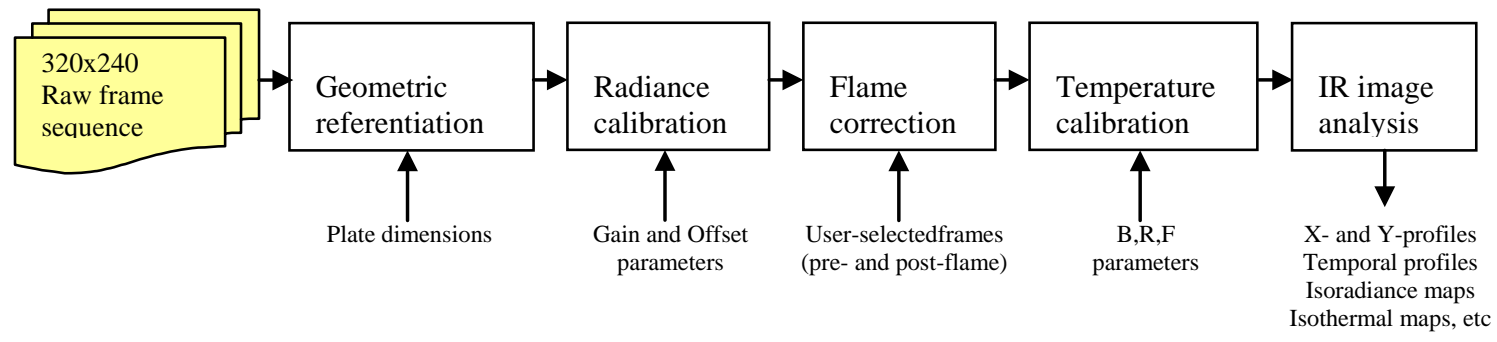

Fig. 2. Image processing scheme.

\section{Measurement method} be written as

When an IR camera images the plate through the flame the measured radiance value $L_{x}$ for a generic frame $X$ can

$$
L_{X}=\tau_{X}^{\text {flame }} \cdot L_{X}^{\text {plate }}+L_{X}^{\text {flame }}
$$

The flame has two effects on the radiation that reaches the camera: to reduce it, due to absorption (described by $\tau_{X}^{\text {flame }}$ ), and to increase it, due to emission (accounted for by the $\tau_{X}^{\text {flame }}$ term). Both effects disappear for a perfectly transparent flame, because $\tau_{X}^{\text {flame }}=1$ implies that $\varepsilon_{X}^{\text {flame }}=0$ and thus $L_{X}^{\text {flame }}=0$. For a real flame, the radiance at the camera will be larger or smaller than the radiance emitted by the plate depending on the relative temperatures of flame and plate. These in turn will depend on the plate region, and therefore flame correction must be different at each point: $\tau_{X}{ }^{\text {flame }}$ and $L_{x}{ }^{\text {flame }}$ will be images rather than numbers. For flames not very thick, as that of our burner, and outside the spectral bands of emission of $\mathrm{CO}_{2}$ and $\mathrm{H}_{2} \mathrm{O}$, it is to be expected that flame transmittance be high, and the main correction will be due to flame emission rather than absorption. Thus, our first task will be to determine the "flame image" $L_{x}{ }^{\text {flame }}$. 
In a typical fire resistance test, when the burner is turned on the radiance measured at a plate point increases sharply (figure 3). The rate of increase slows down until, after a few minutes, a stationary state is reached. When the burner is turned off, radiance shows a sharp fall followed by a gradual cooling. It is clear that the sharp steps at on/off are due to the fast appearance/disappearance of the flame, and it is to be expected that their magnitude will provide an estimation of the flame contribution to radiance.

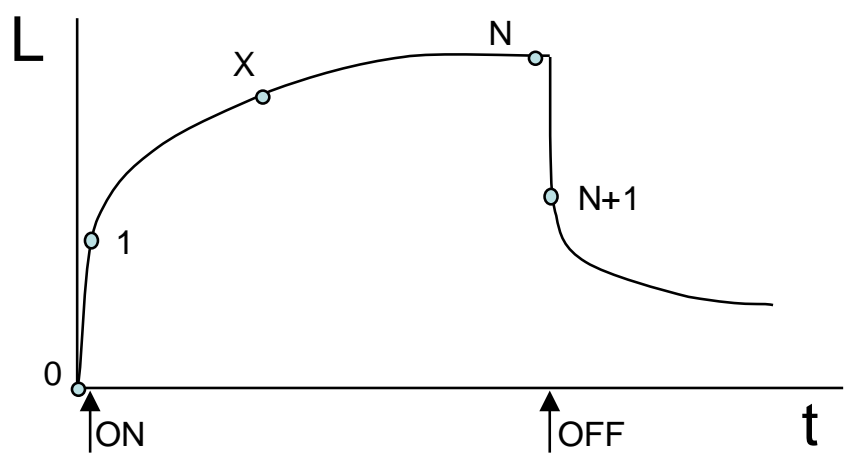

Fig. 3. Schematic time profile of measured radiance versus time for a plate point in a typical fire resistance test. Values for specific frames $(0,1, \ldots X, \ldots N, N+1, \ldots)$ are indicated.

In our setup, the flame takes $\sim 0.1 \mathrm{~s}$ to be fully developed or extinct. Therefore the time between consecutive frames at on/off was fixed at $0.2 \mathrm{~s}$ in order that the flame be completely turned on between frames 0 and 1 , and completely turned off between frames $\mathrm{N}$ and $\mathrm{N}+1$ (in fact, more frames were acquired but were decimated prior to processing).

According to this we can write, at the turning on of the burner,

$$
\begin{gathered}
L_{0}=L_{0}^{\text {plate }} \\
L_{1}=\tau_{1}^{\text {flame }} \cdot L_{1}^{\text {plate }}+L_{1}^{\text {flame }}
\end{gathered}
$$

The radiance increase at the beginning of the test is:

$$
L_{\text {appINI }}^{\text {flame }} \equiv L_{1}-L_{0}=L_{1}^{\text {flame }}-L_{0}^{\text {plate }}+\tau_{1}^{\text {flame }} \cdot L_{1}^{\text {plate }}
$$

If plate heating is slow as compared to the rate of frame acquisition (so that $L_{1}^{\text {plate }} \approx L_{0}^{\text {plate }}$ ) and the flame is thin $\left(\tau_{1}^{\text {flame }} \approx 1\right), L_{\text {appINI }}^{\text {flame }}$ provides an approximation to the initial flame contribution $L_{1}^{\text {flame }}$ because the two terms $-L_{0}^{\text {plate }}$ and $\tau_{1}^{\text {flame }} \cdot L_{1}^{\text {plate }}$ approximately cancel.

Now at each frame $X$ we can obtain an approximate expression for the radiance emitted by the solid plate, by subtracting the flame image (3) from the measured radiance (1):

$$
L_{\text {XappINI }}^{\text {plate }} \equiv L_{X}-L_{\text {appINI }}^{\text {flame }}=\tau_{X}^{\text {flame }} \cdot L_{X}^{\text {plate }}+L_{X}^{\text {flame }}-L_{1}^{\text {flame }}+L_{0}^{\text {plate }}-\tau_{1}^{\text {flame }} \cdot L_{1}^{\text {plate }}
$$

In a similar way, another approximation to $L_{1}^{\text {flame }}$ can be obtained from frames at the turning off: $L_{\text {appFIN }}^{\text {flame }} \equiv L_{N}-L_{N+1}$ and it is found that:

$$
L_{\text {XappFIN }}^{\text {plate }} \equiv L_{X}-L_{\text {appFIN }}^{\text {flame }}=\tau_{X}^{\text {flame }} \cdot L_{X}^{\text {plate }}+L_{X}^{\text {flame }}-L_{N}^{\text {flame }}+L_{N+1}^{\text {plate }}-\tau_{N}^{\text {flame }} \cdot L_{N}^{\text {plate }}
$$

These "initial" and "final" approximations to plate radiance, $L_{\text {XappINI }}^{\text {plate }}$ and $L_{\text {XappFIN }}^{\text {plate }}$ will be accurate provided the following assumptions hold:

(a) The flame has a very large transmittance (i.e., $\tau_{X}^{\text {flame }} \approx \tau_{1}^{\text {flame }} \approx \tau_{N}^{\text {flame }} \approx 1$ )

(b) The flame is perfectly stable (i.e., $L_{X}^{\text {flame }} \approx L_{1}^{\text {flame }} \approx L_{N}^{\text {flame }}$ ) 
(c) The plate has a very large thermal inertia; i.e., its characteristic heating/cooling time is very long as compared to the time between frames. If we define the on/off steps of plate radiance: $\Delta L_{\text {on }}^{\text {plate }} \equiv L_{1}^{\text {plate }}-L_{0}^{\text {plate }}$, $\Delta L_{\text {off }}^{\text {plate }} \equiv L_{N}^{\text {plate }}-L_{N+1}^{\text {plate }}$, this means that $\Delta L_{\text {on }}^{\text {plate }} \approx \Delta L_{\text {off }}^{\text {plate }} \approx 0$.

Now, if these assumptions hold, (4a) and (4b) reduce to

$$
L_{X}^{\text {plate }} \approx L_{\text {XappINI }}^{\text {plate }} \approx L_{\text {XappFIN }}^{\text {plate }}
$$

Equation (5), together with the definitions of $L_{a p p}^{\text {flame }}$ and $L_{\text {Xapp }}^{\text {plate }}$, embodies our "first-order" approximation to plate radiance (the "zero-order" approximation would be to ignore the flame effect altogether, as in the work of Arakawa and Saito [2]). The result, expressed in terms of directly measured quantities, is simply:

$$
L_{X}^{\text {plate }} \approx L_{X}-\left(L_{1}-L_{0}\right) \approx L_{X}-\left(L_{N}-L_{N+1}\right)
$$

Temperature maps can be obtained from radiance images using the calibration function adequate to the spectral range of the camera and an appropriate emissivity value for the plate.
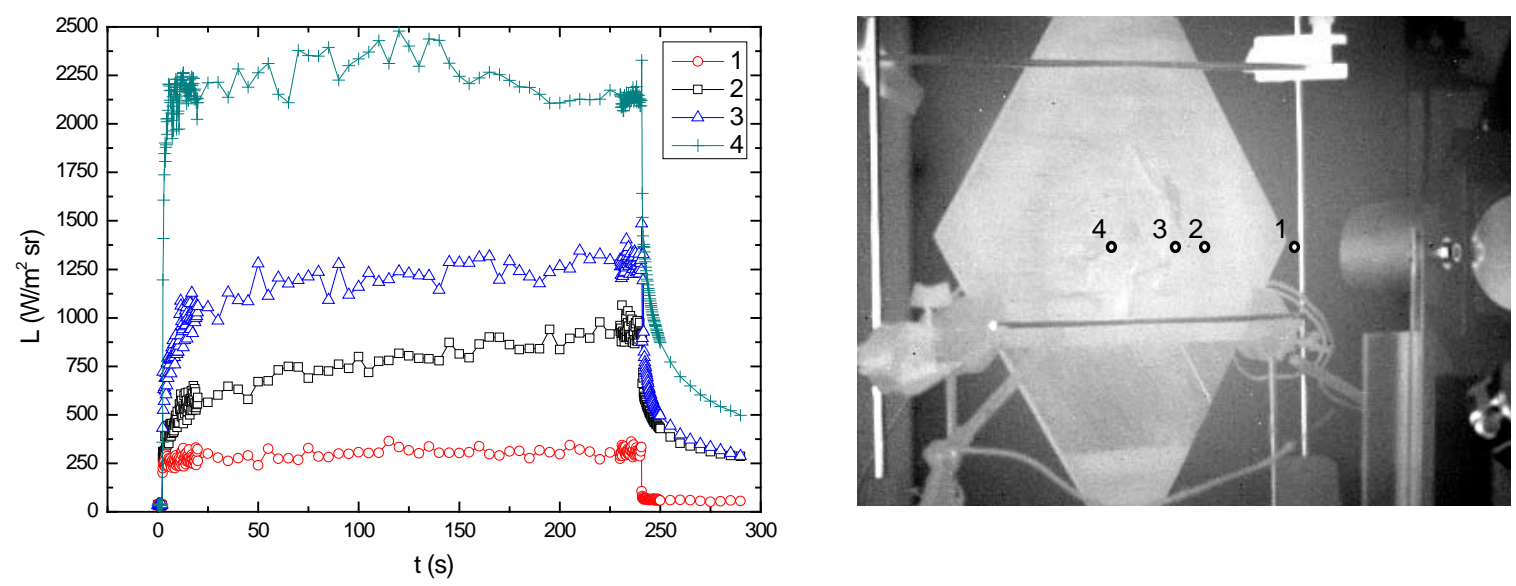

Fig. 4. Left: radiance measured as a function of time at four points during a fire resistance test. Acquisition frequency was 5 FPS (frames per second) during the turning on/off stages (around $t=0 \mathrm{~s}$ and $t=240 \mathrm{~s}$ ), and 0.2 FPS elsewhere (regions of larger acquisition frequency appear to have larger noise). Right: location of the points on an IR image prior to the beginning of the test.

Figure 4 shows radiance data obtained in a typical test at four different points: point 1 is on the flame, with cold background, and points 2, 3 and 4 are on the plate, as indicated on the right-hand panel of the figure. Time between consecutive frames was $5 \mathrm{~s}$, except near the turning on/off of the flame, where it was only $0.2 \mathrm{~s}$ (this is the explanation of the apparent larger noise in these regions). A simple inspection of these data confirms qualitatively assumptions (a), (b) and (c): (a) the low value of radiance at point 1 as compared to the other points is indicative of low flame emissivity, and hence, high transmittance; (b) flame radiance (point 1) is approximately constant and (c) the radiance increase/decrease at on/off are quite sharp at all points.

In order to quantify the errors of the "final" and "initial" approximations, a simple numerical simulation of the test has been performed (to be published in [6]), where the values of parameters in eq. (4) have been estimated from measurements (in the case of flame transmittance, using the emission-transmission method [7]). This simulation shows that the "final" approximation is not sensitive to flame transmittance when the steady state has been reached and that $L_{\text {XappINI }}^{\text {plate }}<L_{\text {XappFIN }}^{\text {plate }}<L_{X}^{\text {plate }}$ except at the beginning of the test. For points 2, 3 and 4 in figure 4 , radiance errors at the steady state for the "final" approximation amount, when translated to temperature, to about 3 to 5 Kelvin, whereas errors in the "initial" approximation are larger by a factor that goes from 4 to 16 . These results imply that the "final" approximation is the better in most cases, and from now on we will use it. 


\section{Results and discussion}

An example of the temperature maps obtained is shown in figure 5. On the left-hand side, the "final" flame georeferenced image is displayed. The colour palette ranges from $0^{\circ} \mathrm{C}$ (black) to $285^{\circ} \mathrm{C}$ (white). These are brightness temperatures, whose low values are to be expected in view of the small emissivity of the flame. The right-hand side shows a plate temperature map corresponding to a few seconds prior to turning off the flame. The colour palette ranges from $0^{\circ} \mathrm{C}$ (black) to $710^{\circ} \mathrm{C}$ (white). The dark horizontal line is a thermocouple (TC); two cold areas in the right image placed symmetrically at the bottom border are due to the plate holders.
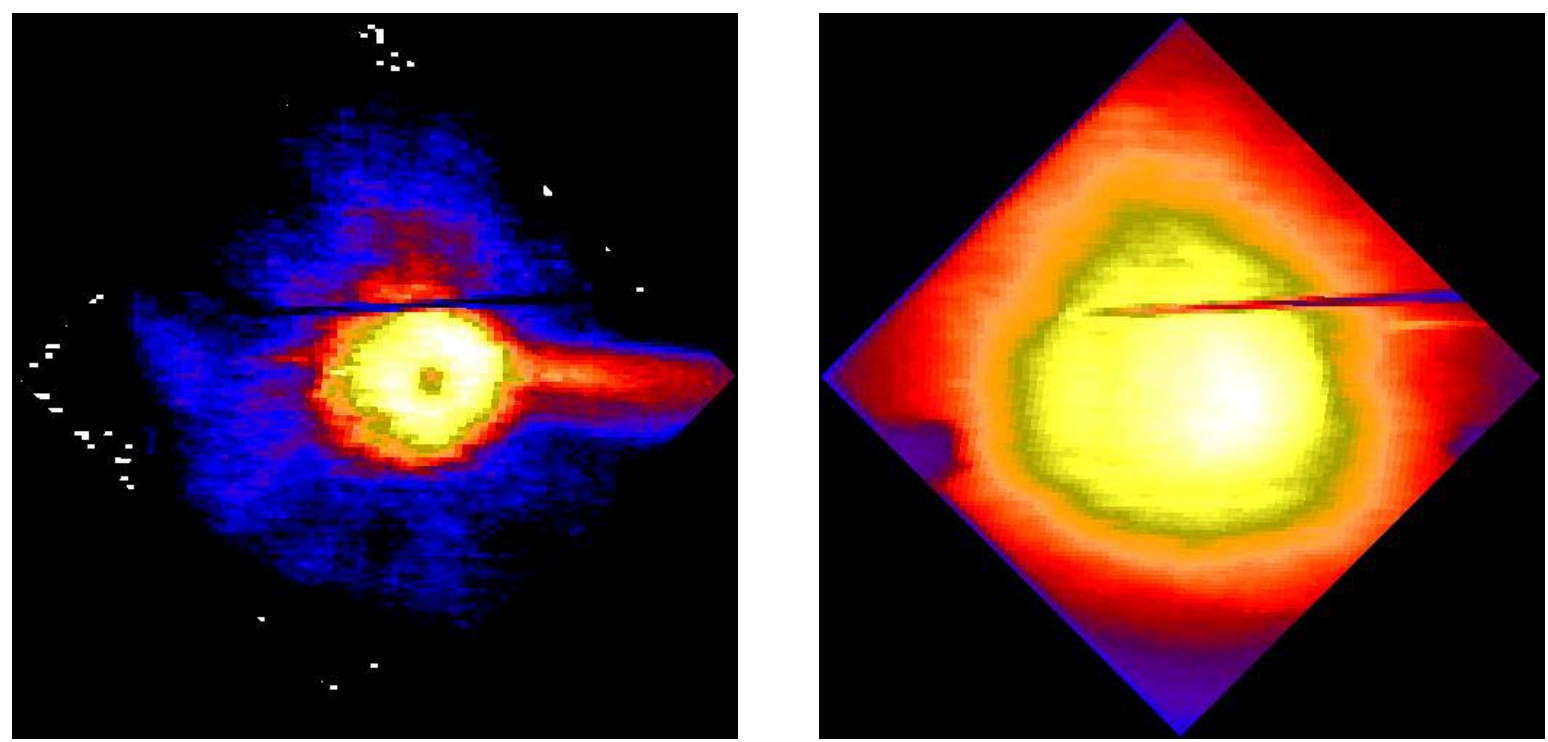

Fig. 5. Left: Geo-referenced "Final" flame temperature image. Colour palette ranges from $0^{\circ} \mathrm{C}$ (black) to $285^{\circ} \mathrm{C}$ (white). Right: Geo-referenced plate temperature image (flame already subtracted), a few seconds before turning off the burner. Colour palette ranges from $0^{\circ} \mathrm{C}$ (black) to $710^{\circ} \mathrm{C}$ (white).
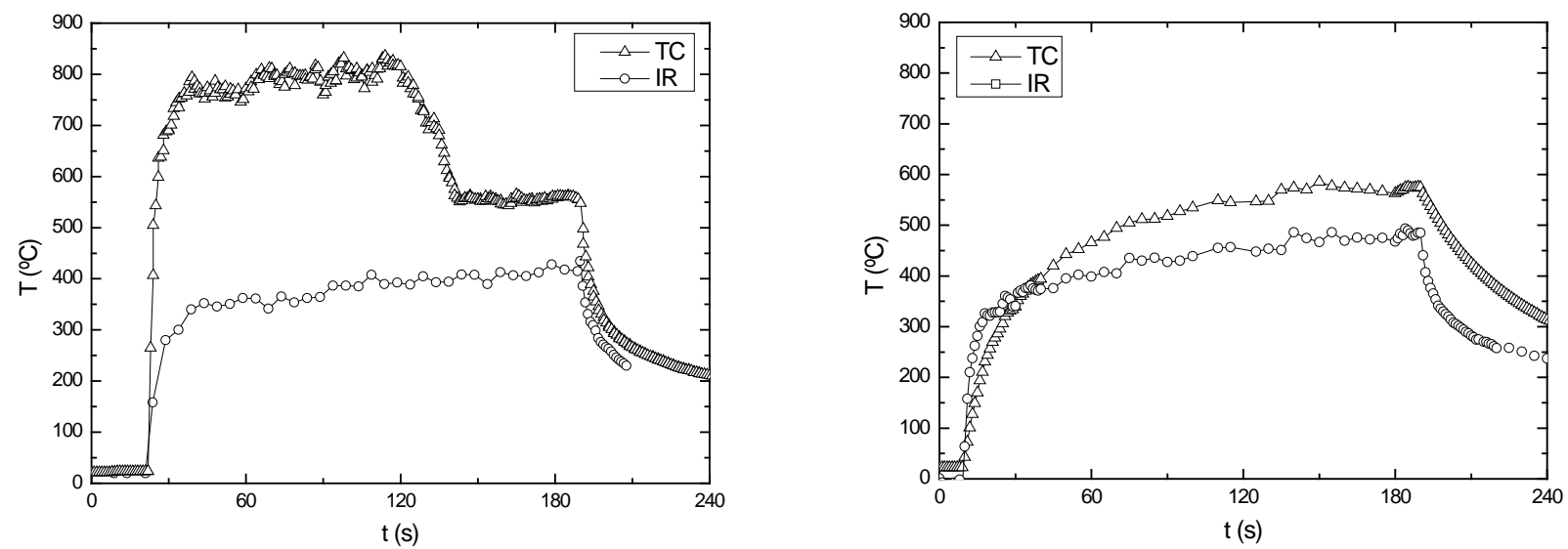

Fig. 6. Plate temperatures measured by thermocouples (triangles) and calculated for the same point with the "final" IR method (circles) with emissivity $\varepsilon=1$. On the left, a thermocouple with a $\varnothing 1.5 \mathrm{~mm}$ flexible shield was used. A sharp fall at 120-140 s is apparent, caused by displacement of the junction due to the air flow generated by the blower. On the right, a thermocouple with a rigid $\varnothing 6.0 \mathrm{~mm}$ shield was used. Response time is much slower, but the junction position is stable and there are no sharp steps in thermocouple data. 
In an effort to validate the method, comparisons at specific points have been made with TC readings, which are the standard method for surface temperature measurements in fire resistance tests [8].

Figure 6 (left) compares two typical temperature time profiles for the same point, measured with K-type thermocouples with a $\varnothing 1.5 \mathrm{~mm}$ flexible shield (triangles, marked as TC) and with the infrared "final" method (circles, marked as IR). There are two salient features: first, TC temperatures show a large step decrease between 120 and 140 s; second, TC temperatures are larger than IR temperatures.

These large step variations in TC temperatures were common in our first experiments. It was later realized they were a consequence of small displacements of the TC junction, which, as a result of the turbulent motion of flame gases, moved into the boundary layer (thus decreasing the measured temperature) or out of it (increasing the temperature).

The original TCs were replaced by more robust ones, with a rigid $\varnothing 6.0 \mathrm{~mm}$ shield diameter and a new setup that assured good contact with the plate (as seen in figure 1). A typical result with this modified setup is shown on the right hand side of figure 6. As expected, the step variations disappeared. The new, bulkier TCs show a much longer thermal inertia, but at the saturation stage, TC temperatures remain larger than IR temperatures. The most obvious explanation to this error is plate emissivity: since IR temperatures in figure $6 \mathrm{~b}$ have been obtained with $\varepsilon=1$, the real temperatures must be larger. However, this is not enough to account for the temperature difference. For instance, in order for the IR temperatures to reach the TC values at the saturation stage in figure 6 (right), a value of $\varepsilon=0.75$ has to be used. This is much too low, since, as previously explained, emissivity for an undamaged surface of CFRP is $\varepsilon \approx 0.80$, and in our case, due to previous burnout, a reasonable value is $\varepsilon \approx 0.90$ : in all cases, in order to account for difference between TC and IR temperatures emissivity should have a value too low.
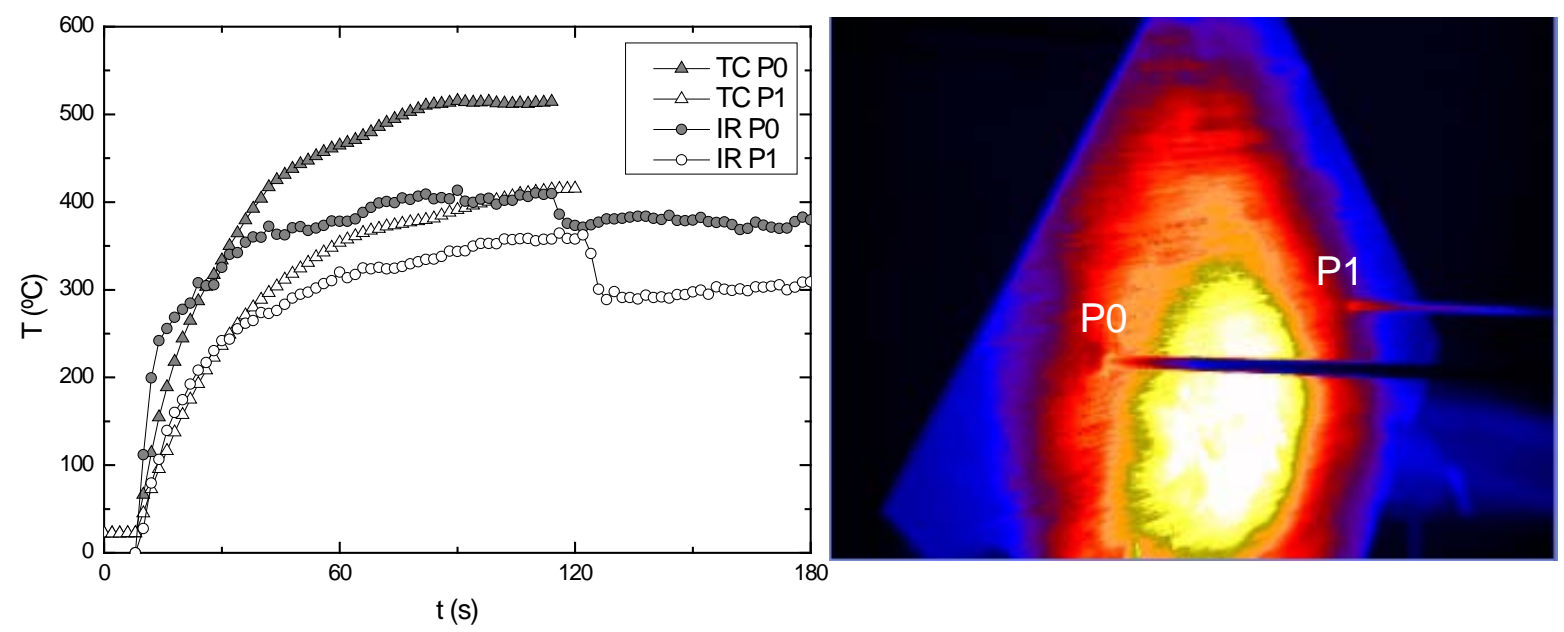

Fig. 7. Left: Plate temperatures for two different points, $P 0$ (grey symbols) and P1 (white symbols), measured by thermocouples (triangles) and calculated for the same point with the "final" IR method (circles) with emissivity $\varepsilon=1$. The TCs are withdrawn at $t=114 \mathrm{~s}$ (point $P 0$ ) and at $t=120 \mathrm{~s}$ (point P1). A drop in IR temperatures appears when the TC loses contact with the plate. Right: position of the points $P O$ and $P 1$ on the plate. It can be appreciated that the points where the TC make contact with the plate are hot spots relative to their neighborhood.

This seems to point to a systematic error in TC temperatures. A possible explanation is the conduction of heat through the TC shield from the flame to the TC junction. In order to verify this, a specific experiment was performed to test the effect of TCs on the surface temperature. With the camera placed near the plate in order to have good spatial resolution, the TC was withdrawn from the plate while the flame was still on. When the TC lost contact with the plate, brightness temperature at the contact point as measured by the camera actually dropped. Results are shown in figure 7 (left) for two different points, labeled as P0 and P1. The location of the points can be seen in figure 7 (right). Circles stand for the IR final approximation to plate brightness temperatures (i.e., calculated with $\varepsilon=1$ ); triangles stand for TC readings (their graphs have been interrupted after loss of contact, since they are no longer meaningful). In both cases there is a clear drop in IR temperature when the $T C$ loses contact with the plate $\left(\Delta T \approx 30^{\circ} \mathrm{C}\right.$ at $P 0$ and $\Delta T \approx 60^{\circ} \mathrm{C}$ at $\left.P 1\right)$. In fact, the observation of the IR image shows that contact points are hot spots on the plate surface (figure 7 , right). 
It is well known that TCs, being intrusive probes, are subject to systematic errors when measuring flame temperatures. In particular, flame quenching in the vicinity of the TC, resulting in measuring temperatures lower than real, has been often observed [7]. Our results demonstrate that in the present case, the error induced by the TCs is very significant, but has the opposite sign, and is very probably related to heat conduction in the TC shield through the boundary layer from the flame to the plate. This error is very difficult to minimize, since using thinner TC implies that no stable contact with the plate is achieved within the turbulent flame environment. In addition, due to the large spatial heterogeneity of flame temperatures, there is no spatial configuration of the TCs that avoids heat conduction through their shields to the plate.

These systematic errors in TC measurements imply that the advantage of the IR technique lies not only in providing fast two-dimensional measurements (temperature maps) without previous sample preparation, but also in the possibility of giving more accurate values of temperature. With the method here developed to subtract flame effect, the main uncertainty that remains is the value of plate emissivity, but even relatively large errors in emissivity may give errors in temperature that are smaller than those due to TC effects. As an example, a brightness temperature of $410^{\circ} \mathrm{C}$ (as in P0 in figure 7 , just before the TC is retired) corresponds to a real temperature of $423^{\circ} \mathrm{C}$ if $\varepsilon=0.95$ and of $453^{\circ} \mathrm{C}$ if $\varepsilon=0.85$; i.e., an uncertainty of \pm 0.05 in $\varepsilon$ translates into $\pm 15^{\circ} \mathrm{C}$ in temperature. In any case, the uncertainty in $\varepsilon$ and the corresponding error in temperature can be greatly reduced if the plate emissivity is measured.

\section{Conclusions}

In this work an infrared method for the measurement of the temperature of solid objects exposed to fire has been developed and applied to composite plates undergoing a fire resistance test. The IR method discounts the flame effect and provides sequences of two dimensional temperature maps of the plate during the whole test, with no need of previous sample preparation, in a non-intrusive way, and with good time resolution.

The method is based in the subtraction of a "flame image" that can be formed out of the IR images of the plate when the flame is turned on ("initial" approximation) or off ("final" approximation). The method is accurate for flames that are thin, stable, and that are turned on/off fast as compared to the thermal time constant of the plate. The validity of the method for our specific setup has been analyzed in experimental tests. It has been found that systematic errors due to the intrusive nature of thermocouples are very large and difficult to avoid, whereas errors in the "final" IR approximation are much smaller for the stationary regime of the test if emissivity is known, and remain relatively low even for moderate uncertainties in emissivity.

\section{REFERENCES}

[1] Meléndez J., Aranda J. M., de Castro A. J. ,and F. López., "Measurement of forest fire parameters with multispectral imaging in the medium infrared", QIRT Journal; vol. 3, pp 183-200 (2006)

[2] Arakawa A., Saito K., and Gruver W. A., "Automated Infrared Imaging Temperature Measurement with Application to Upward Flame Spread Studies", Combustion and Flame; Vol: 92, Issue 3, pp222-230 (1993)

[3] Pilch N.D, Pettegrew R.D., and Ferkul P., "Quantitative surface emissivity and temperature measurements of a burning solid fuel accompanied by soot formation", AIAA, Aerospace Sciences Meeting and Exhibit, 39th, Reno, NV; USA; 8-11 Jan. 2001 (2001).

[4] Wolfe W. L. and Zissis G. J., The infrared handbook (Arlington, VA: Office of Naval Research, 1978)

[5] Starnes M. A., Carino N. J. and Kausel E. A., "Preliminary Thermography Studies for Quality Control of Concrete Structures Strengthened with Fiber-Reinforced Polymer Composites", J. Mat. in Civ. Engrg. 15 266-273 (2003)

[6] Meléndez J., Foronda A., Aranda J. M., López F. and López del Cerro F. J., "Infrared thermography of solid surfaces under fire", to be published in Measurement Science and Technology (2010)

[7] Aranda J.M., Briz S., Meléndez J., de Castro A. J., and López F., "Flame analysis by IR thermography and IR hyperspectral imaging", Proceedings of QIRT 2000, D. Balageas, J.L. Beaudoin, G. Busse, G.M. Carlomagno eds., Reims, France (2000).

[8] EGOLF (The European Group of Official Laboratories for Fire Testing) 2000 Procedures for the choice, use, checking, mounting and replacement of instrumentation used for temperature measurement in fire resistance tests Ref No EGOLF / TR / 2:2000 http://www.egolf.org.uk/FORUMATTACHMENTS/762/tr2_2000.pdf 